\title{
RECOMENDACIONES PARA LA EVALUACIÓN DE LA ACTIVIDAD FÍSICA EN POBLACIÓN INFANTIL
}

Carlos Álvarez Bogantes, Dr.

Escuela Ciencias del Deporte,

Universidad Nacional,

Costa Rica.

calvarez@una.ac.cr

RESUMEN

El propósito de este artículo fue el de revisar los patrones de movimiento de los niños y las niñas y como estos pueden impactar la evaluación de la actividad física. Para el logro de la evaluación de esta se requiere de instrumentos que sean sensibles para que se detecte, codifique o registre la actividad física esporádica e intermitente de los niños y las niñas, sin olvidar las regulaciones que existen en el nivel científico. Varios de los instrumentos de más uso son: el autorreporte, la observación directa y el monitoreo de la frecuencia cardiaca. Los autorreportes requieren de habilidades de pensamiento abstracto y de buena memoria, lo que en edades tempranas no se ha desarrollado, por otro lado, la observación directa y el monitorio cardiaco, requieren de gran cantidad de tiempo y de alta tecnología para su aplicación, respectivamente. La recomendación más ampliamente extendida, es que se recurra a una combinación instrumentos, para garantizar la mayor cantidad de información y de validez de la investigación.

PALABRAS CLAVES: autorreportes, patrones de movimiento, monitores cardiacos, actividad física y observación.

\section{RECOMMENDATIONS FOR THE ASSESSMENT OF PHYSICAL ACTIVITY IN THE INFANTILE POPULATION}

\section{ABSTRACT}

The purpose of this article was to review movement patterns of children and how they may impact the assessment of physical activity. To accurately assess these patterns, sensitive instruments are required to detect, code, or record children's sporadic and intermittent physical activity, also taking into consideration scientific regulations. Some of the most widely used instruments are: self-reports, direct observation, and heart monitoring. Self-reports require abstract thinking and good memory, abilities that have not yet been developed in the early years. On the other hand, direct observation and heart monitoring are more time consuming and require advanced technology, respectively. The most accepted recommendation is combining instruments to ensure greater quantity of information and more validity in the research.

KEY WORDS: self-reports, movement patterns, heart monitors, physical activity, and observation.

\section{INTRODUCCIÓN}

La medición de la actividad física en los niños y las niñas es una empresa importante y retadora, debido a que esta población sufre cada vez más los efectos de patrones de vida sedentarios, propiciados no sólo por condiciones tecnológicas que estimulan estilos de vida inapropiados, sino también, por una cultura de inmovilidad, que eventualmente podría ponerlos en mayor riesgo de enfermedades degenerativas (Rowland, 1990; Shephard, 1994). Los estudios más importantes han 
mostrado que los niños y las niñas más activos (as), presentan mejores niveles de aptitud física para la salud (Bar-Or, 1982; Sallis, Buono, Roby, Micale, \& Nelson, 1993), presiones sanguíneas más bajas, niveles más bajos de HDL-colesterol y de grasa corporal (Freedson et al, 1989; U.S. Department of Health and Human Services, 1996), lo que disminuye el riesgo de enfermedades cardiodegenerativas para este grupo. Sin embargo, los beneficios de la actividad física en la prevención de estas enfermedades en los niños y las niñas, no se han establecido contundentemente, debido a que las conductas inapropiadas para la salud toman tiempo para que causen los efectos nocivos en el organismo o para que influencien la aparición de enfermedades crónicas, las cuales son desequilibrios orgánicos que se manifiestan en la vida adulta, en la mayoría de los casos (U.S. Department of Health and Human Services, 1996).

Algunos estudios reportan una relación positiva moderada entre inactividad física y los factores de riesgo coronario (Welk \& Wood, 2000); pero otros han reportado resultados no significativos estadísticamente. En el presente, sigue creciendo la información relacionada con el proceso de inicio de aterosclerosis en edades más tempranas, lo que aumenta la importancia de la actividad física en el entorno escolar para poder impactar con mayor éxito los hábitos saludables de los niños y las niñas. Aunado a lo anterior, la evidencia también muestra que los factores de riesgo coronario (hiperlipidemias, sedentarismo, obesidad y fumado) que propician la aterosclerosis y la enfermedad coronaria, se extienden dentro de la vida adulta (Harrel et al, 1998), aumentando las probabilidades del desarrollo de las enfermedades cardiodegenerativas. Gran variedad de estudios han mostrado la efectividad del uso de la actividad física en la intervención en los niños y las niñas con obesidad, en la disminución de factores de riesgo de enfermedades degenerativas, en el tratamiento de la diabetes mellitus y en la disminución de niveles de sedentarismo en los (as) jóvenes (Eptein, 1984; Mahoney, Lauer, Lee, \& Clarke et al, 1991; Whitaker, Wright, Pepe, Seidel \& Dietz, 1997; Rippe \& Hess, 1998; Pate et al, 1999). No obstante, la medición de esta variable en la niñez presenta limitaciones metodológicas, que dificultan la utilización de formas no invasivas y exactas, a la hora de realizar las medidas. Esto hace necesario desarrollar medidas de la actividad física que sean prácticas, de bajo costo y exactas, que aseguren resultados más confiables y válidos.

Muchos métodos y técnicas para medir el movimiento corporal han sido reportados para ser usados en población adulta. Welk y Wood (2000) revisaron infinidad de instrumentos, incluyendo autorreportes, observaciones directas, instrumentos mecánicos y electrónicos, monitores de frecuencia cardiaca, calorimetría indirecta y otros. Cada una de las formas mencionadas tiene sus debilidades y puntos fuertes, que deben ser considerados a la hora de seleccionar una de las opciones anteriores. No existe instrumento o metodología que sirva para todos los objetivos (Pate, 1993; Wareham et al, 1998).

El propósito de este artículo, es el de enfocarse en las diferentes formas de medir la actividad física de los niños y las niñas, y recomendar las mejores opciones, bajo las características particulares de esta población. Aunque algunos de los temas o enfoques que se mencionarán serán aplicables a todas las poblaciones, el mayor esfuerzo recaerá en la población escolar.

\section{DESARROLLO}

Métodos para la medición de la actividad física en los niños y las niñas.

A la hora de escoger el método para determinar la actividad física en los niños y las niñas, se requiere que ese término quede bien definido, pues este se refiere a cualquier movimiento del cuerpo producido por los músculos esqueléticos que resulta en un determinado gasto calórico (Caspersen et al, 1985). En la práctica investigativa, la definición operacional de la actividad física depende en cómo es medida y registrada. Las variables de frecuencia, intensidad y duración son comúnmente usadas para caracterizar los patrones de movimiento; otra variable de interés es el 
gasto calórico. Para los fines de este artículo se han escogido cuestionarios, monitores cardiacos y sensores de movimiento como las formas más apropiadas para la medición de los patrones de movimiento en los niños y las niñas (Welk et al, 2000).

\section{Autorreportes o cuestionarios autoaplicados}

Los cuestionarios continúan siendo la forma más usada al medir la actividad física. Sin embargo, es importante tratar de mencionar sus fortalezas y debilidades con el objeto de determinar la conveniencia de usarlos con los niños y las niñas. En el presente trabajo los autoreportes pueden ser cuestionarios autoadministrados, recordatorios de 24 horas o las entrevistas. Los beneficios reconocidos de los autoreportes comprenden la facilidad para obtener la información cuando se trabaja con grupos grandes, a un costo reducido. Los cuestionarios y autoreportes no alteran la conducta que se estudia y posibilitan el estudio de todas las dimensiones de la actividad física (Sallis, et al, 2000; Pancrazi et al, 2000; Welk et al, 2000). Estos han sido usados con amplios rangos de edad, propiciando que las mediciones puedan ser adaptadas para calzar las necesidades de una población específica o a una pregunta de investigación. Cuando se discuten las limitaciones de los autoreportes o cuestionarios de autoaplicados, autores como Ainseworth et al (1994), Warnecke et al (1997) advierten que especialmente los que requieren recordar la actividad física, necesitan destrezas cognitivas complejas. Los niños, las niñas y los adultos mayores están propensos a tener limitaciones con la memoria a la hora de recordar y registrar la información requerida, lo que limita el uso de estos instrumentos en estas poblaciones. Adicionalmente, es importante tomar en cuenta a la hora de utilizar los cuestionarios o autoreportes la aclaración de términos que son ambiguos en su naturaleza como actividad física, moderada intensidad y tiempo libre.

Otros estudios han utilizado instrumentos de reportes autoaplicados (Craig et al, 1996; Gortmaker et al, 1999), que han reportado niveles de actividad física superiores a los reportados usando monitores cardiacos o técnicas de observación. Algunos autores, como Welk (2000) y Pate et al (1996) sospechan que usando los autoreportes o cuestionarios se podría presentar una sobreestimación de los niveles de actividad física en los niños y las niñas. Ellos argumentan que una percepción exagerada del tiempo y el esfuerzo podría causar que los resultados obtenidos se alteren con los auto reportes.

Los instrumentos de autoreporte en los (as) jóvenes, en los niños y las niñas evidencian un gran auge, como lo puede indicar el encontrar 17 referencias, que han demostrado relativa validez y confiabilidad. Sin embargo, esta cantidad de literatura es solo una reproducción de mediciones, en vez de sistematizar la exploración y medición en todos los rangos de edades de los niños, las niñas y los jóvenes. Sallis (1991) ha reportado solo una correlación moderada entre varias formas de autoreportes y otros criterios objetivos. La carencia de una correspondencia fuerte y la tendencia descrita de la sobreestimación en estos instrumentos ha conducido al consenso de que los niños y las niñas no pueden proveer información exacta de sus patrones de movimiento.

Dentro de las formas mencionadas en este artículo de autoreportes la mayoría de autores concuerdan en que los recordatorios de 24 horas muestran las mejores posibilidades en los niños y las niñas (Pate, 1993; Sallis et al, 1993; Sallis, 1991). El punto fuerte de este recordatorio es que es más fácil recordar lo del día anterior que lo de una semana, así es como valores de intensidad, frecuencia y duración son fácilmente recordadas. Una de las debilidades que se le achacan es que la información debe ser obtenida en diferentes días para tomar en cuenta la variabilidad intraindividual en los patrones de actividad. (Welk et al, 2000). Para evitar este tipo de error algunos investigadores recomiendan registrar los bloques de actividad de los participantes en lugar del tiempo (Trost et al, 1996; Weston, Petosa \& Pate, 1997). También, Going et al (1999) recomiendan convertir la información en un índice de actividad que incorpore las variables de intensidad y duración. 
Un cuestionario de 24 horas que ha sido aceptado es el Recordatorio del Día Previo de Actividad Física (Weston et al, 1997). Este recordatorio enfatiza las actividades realizadas antes y después de la escuela en bloques de 30 minutos, por lo que se considera una herramienta apropiada para determinar la cantidad de actividad que los niños y las niñas ejecutan durante su tiempo libre. Observando la actividad física realizada y las conductas sedentarias ejecutadas, se puede desarrollar una intervención para incrementar los momentos de actividad física y disminuir el sedentarismo (Eptein et al, 1995; Eptein et al, 1997). Aunque los recordatorios de 24 horas son los que están en boga, los que se realizan sobre un periodo de tiempo de una semana, se consideran más representativos. Este formato asume que la semana previa es representativa de la actividad del niño y la niña en otras semanas. Sin embargo, tiene las debilidades de confiar en la memoria y en que ellos mantienen una conducta de movimiento similar a través del año, lo que ignora los cambios producidos por el clima en los patrones de movimiento a estas edades (Welk et al, 2000).

Uno de los recordatorios más usados a la hora de medirla es el Cuestionario de Actividad Física para Niños (Crocker et al, 1997; Kowalski et al, 1997), el cuál fue diseñado para evaluar la actividad en los siete días previos, pero también puede ser usado para constatar los niveles típicos de actividad física. Si el objetivo es el de describir los hábitos generales de los niños y las niñas, algunas formas alternativas que no utilizan la frecuencia, intensidad y duración de la actividad pueden usarse, con el objeto de discriminar entre la población activa y la sedentaria (Crocker, et al, 1997).

Independientemente de la forma que se utilice para recolectar la información, los resultados pueden ser mejorados, desarrollando nuevas formas de capacitación en los procedimientos para completar los cuestionarios o autoreportes. Para finalizar este apartado se puede decir que hay una cantidad de autoreportes para medir la actividad física en niños y en niñas con la adecuada confiabilidad, con la validez de contenido apropiada, que pueden ser usados. Se encontró una deficiencia en los autoreportes que determinan fuerza, flexibilidad u otras dimensiones de la aptitud física para la salud.

\section{Sensores de movimiento}

Los sensores son instrumentos mecánicos y electrónicos que detectan movimiento y aceleraciones de una extremidad o el tronco, dependiendo en donde el aparato es sujetado. En la actualidad hay diferentes tipos de aparatos que difieren en complejidad y costo, que van desde los podómetros hasta los más sofisticados acelerómetros (Basset, 2000).

Gran cantidad de estudios han examinado la confiabilidad y validez de estos aparatos en los niños y en las niñas, en condiciones de laboratorio y en el campo (Eston et al, 1998; Welk \& Corbin, 1995; Trost et al, 1998). Similar a los estudios con los adultos, el consenso general es que los sensores de movimiento brindan medidas válidas de la actividad física, pero resultados relativamente cuestionables del gasto energético. Una de las limitaciones que presentan los sensores es la imposibilidad de evaluar el tren superior del sujeto cuando realiza actividades de lanzar, agarrar, levantar, halar y otras de la vida diaria que incluyen movimiento del tren superior. Bouten et al (1997) han reportado que los sensores de movimiento al ser usados para valorar la actividad física en la vida cotidiana ofrecen resultados menores de gasto calórico. Aunque investigaciones similares no han sido realizadas en los niños y en las niñas, se sospecha que lo mismo pasaría al evaluar la actividad intermitente a estas edades. Cuando se usan los sensores de tres dimensiones se cree que estos ofrecen ventajas sobre los de una dimensión, esto no ha sido corroborado en las investigaciones consultadas. Para evitar errores en las estimaciones del gasto energético, Welk et al (2000) recomiendan reportar los pasos o los minutos de actividad. Uno de los acercamientos más comunes en la investigación de campo usando sensores es usar puntos de quiebra para determinar la cantidad de tiempo gastado en diferentes categorías de intensidad (Janz, 1994). Aunque se pudiera 
pensar que esta es una estrategia apropiada, se debe tomar en cuenta que esta produciría reportes de gasto calórico inferiores en los niños, se basan en el número de cuentas registradas durante un episodio continuo de actividad. Debido a que la mayoría de acelerómetros usan un procedimiento integrado para resumir las cuentas de los movimientos, el valor al final del minuto refleja el total de cuentas del tiempo reportado, esto hace que periodos cortos de alta intensidad sean ignorados por los periodos de descanso, cuando se realiza el reporte del minuto (Dale, 1999). Los valores de la actividad en esos minutos pueden ser tomados como tiempo inactivo, si los puntos de quiebra están basados en las cuentas que suceden durante el ejercicio continuo. Para evitar este error potencial, los puntos de quiebra deben considerar la actividad intermitente en la conducta de movimiento de los niños y las niñas (Basset, 2000; Welk et al, 2000). Bajo esta perspectiva, técnicas de observación realizadas al mismo tiempo de la utilización del sensor podrían ofrecer el mejor criterio o también se podría procesar la información de los sensores usando intervalos de tiempo menores a un minuto. Aunque las limitaciones podrían desmotivar el uso de los acelerómetros, estos son una de las herramientas más útiles para valorar la actividad física sobre periodos extensos de tiempo. El costo y el tiempo administrativo talvez sería muy alto cuando se realiza con poblaciones muy grandes, pero son muy prácticos para intervenciones pequeñas o validación de otras técnicas (Freedson y Miller, 2000).

\section{Monitores cardiacos}

Bajo condiciones controladas de laboratorio, la actividad física, la frecuencia cardiaca y el consumo máximo de oxígeno están altamente relacionadas y muestran una relación lineal, especialmente entre frecuencias cardiacas de 110 a 140 Consecuentemente, el uso de la frecuencia cardiaca como un marcador fisiológico del consumo de oxígeno es una aproximación altamente apropiada para evaluar la actividad física (Patterson, 2000).

En niños y en niñas, los monitores cardiacos proveen un indicador objetivo de los efectos fisiológicos de la actividad física, brindando medidas válidas de la frecuencia cardiaca en esta población (DuRant et al, 1993). En la actualidad los monitores para determinar la frecuencia cardiaca han alcanzado un desarrollo tecnológico envidiable, lo que los hace no solo confiables y válidas las mediciones, pero también fáciles de realizar, por ser equipos más que portátiles y de un uso simple. Con este tipo de equipos, los patrones de movimiento pueden ser determinados usando la frecuencia, intensidad y la duración de la actividad física (Welk et al, 2000). Sin embargo, se debe ser cauteloso a la hora de usarlos ya que hay varias fuentes de error en las mediciones, que provienen de factores como la temperatura, humedad y la presión emocional, los cuales causan aumentos en la frecuencia cardiaca que pueden alterar los resultados (Melanson et al, 1996; Montoye, Kemper, Saris, \& Washburn, 1996). Además, la relación entre frecuencia cardiaca y consumo máximo de oxígeno es afectado por la cantidad de masa muscular y el tipo de contracción muscular que se utiliza durante el ejercicio. Otros factores como la deshidratación y la fatiga también causan una disminución en la relación entre frecuencia cardiaca y el consumo de oxígeno (Montoye et al, 1996; Rowland \& Eston, 1997). A la hora de comparar frecuencias cardiacas entre individuos de géneros y aptitud física diferentes se enfrenta con otro error, ya que esta se ve afectada por esas variables. Personas con una condición física pobre comparados con aquellos que tienen una mejor condición tendrán una frecuencia cardiaca mayor en un dado consumo de oxígeno. Los mayores errores en la estimación del gasto calórico se dan en bajas y altas intensidades de la actividad física (Rowland, 1997). La relación entre frecuencia cardiaca y consumo de oxígeno es verdadera a intensidades moderadas, pero podría dejar de ser exacta a bajas y altas, según afirma el autor anterior. Se podría finalizar este apartado diciendo que los monitores de frecuencia cardiaca no son formas perfectas de registrarla, pero eliminan un gran nivel de subjetividad, que caracteriza a otras formas de valorar la actividad física. 
Una de las mejores opciones a la hora de valorar la actividad física en los niños y las niñas es el uso de métodos y técnicas múltiples, debido a que se provee una mejor descripción de la actividad, permitiendo la triangulación de los resultados (Welk et al, 2000).

\section{¿Cuál método o instrumento aplicar?}

A través del tiempo las concepciones equivocadas de la actividad física en edades tempranas, han considerado que los niños y las niñas son adultos pequeños. Sin embargo, esto ha sido aclarado y en la actualidad se reconoce como válido el concepto que ellos son anatómica, fisiológica, y sicológicamente inmaduros, lo que lleva a considerar métodos diferentes de los utilizados con los adultos a la hora de medir el grado de actividad o cuando se requiere determinar el de actividad calórica (ACSM, 1995). Estudios con niños y niñas, han mostrado que ellos y ellas incrementan la frecuencia cardiaca dentro de la zona de entrenamiento considerada para los adultos por periodos de tiempo muy reducidos, lo que hace que en apariencia ellos sean muy inactivos. Corroborando lo anterior, Armstrong y Bray (1991) y Welk (1995) encontraron que el 77\% de los niños y el 88\% de las niñas mostraron ser inactivos, siguiendo los criterios utilizados para calcular el sedentarismo en la población adulta. Lo que estos dos estudios muestran es que en edades tempranas se es activo en diferentes formas a las mostradas por los adultos. Esto hace que a la hora de evaluar el grado de actividad física en la niñez se debe respetar las características que se han mencionado con anterioridad. La necesidad de valorar la actividad física en esta población obedece a la necesidad de evaluar el éxito de determinada intervención o para estimar si la población reúne los criterios para aptitud física para la salud, que han sido establecidos para esa población (Welk, 2000).

A la hora de escoger el método para determinar la actividad física en los niños y en las niñas, se requiere que ese termino quede bien definido, ya que se refiere a cualquier movimiento del cuerpo producido por los músculos esqueléticos que resulta en un determinado gasto calórico (Caspersen et al, 1985). En la práctica investigativa, la definición operacional de la actividad física depende en cómo es medida y registrada. Las variables de frecuencia, intensidad y duración son comúnmente usadas para caracterizar los patrones de movimiento; otra variable de interés es el gasto calórico.

Para los fines de este artículo se escogieron cuestionarios, monitores cardiacos y sensores de movimiento como las formas más apropiadas para la medición de los patrones de movimiento en los niños y las niñas (Welk et al, 2000; Welk et al, 2000). La escogencia de una herramienta para medir la actividad física depende de la aplicación que se vaya a realizar. Estudios de balance energéticos y los de pérdida de peso requieren medidas muy exactas, por lo que solo el método de doble etiquetado de $\mathrm{H}_{2} \mathrm{O}$ es aceptado, aunque este método se tendría que ignorar si lo que se quiere es evaluar la actividad física (Basset, 2000). Si lo que se quiere es el estudio de los patrones de movimiento, no hay nada como los acelerómetros, podómetros, los monitores de frecuencia cardiaca o una combinación de los anteriores. Estos instrumentos tienen la habilidad de almacenar la información en forma permanente. Cuando el investigador lo que desea es determinar el tiempo que se gasta en actividad física moderada o intensa, los cuestionarios pueden ser considerados para tal efecto. Para estudios epidemiológicos, el podómetro es el instrumento por excelencia, debido a su costo y a la simpleza del mismo, pudiéndose combinar con el uso del autoregistro para obtener mejores resultados (Basset, 2000; Freedson et al, 1997, 1998).

\section{CONCLUSIONES}

La naturaleza de los patrones de movimiento de los niños y las niñas debe ser considerada a la hora de seleccionar un instrumento para determinar los cambios que produce un programa de intervención o para determinar los patrones de movimiento. Para que la valoración de estos en edades tempranas pueda ser con una mayor exactitud, un instrumento debe ser suficientemente sensitivo para detectar y registrar la actividad esporádica e intermitente. Además, hay que poner atención no solo a la cantidad de días, pero también al momento del día que se pretende hacer las 
mediciones para seleccionar el instrumento, para que refleje las guías de actividad física recomendadas para los niños y las niñas.

Diferentes métodos e instrumentos han sido descritos para determinar la actividad física en los niños y las niñas. Sin embargo, se recomienda no aferrarse a un método específico, ya que la selección apropiada depende de la pregunta de investigación que está siendo tratada y de la relativa importancia que se le asigne a la exactitud y a la posibilidad de aplicación del método o instrumento. En los niños y las niñas está demostrado que carecen de las destrezas para que realicen registros exactos, cuando se usan autoreportes o recordatorios de 3 o 7 días, por lo que se recomienda su uso en combinación con otros métodos o instrumentos. Sin embargo, este es uno de los formas para evaluar la actividad física en edades tempranas más apropiada cuando se trabaja con muestras grandes. Otro de las formas más populares ha sido los monitores cardiacos, pero se debe tomar en cuenta que las variables ambientales, así como la tensión emocional pueden alterar estas medidas. El costo de estos monitores cardiacos ha limitado su uso a muestras pequeñas. El instrumento más reciente a la hora de determinar la actividad física en los niños y en las niñas ha sido los sensores electrónicos, los cuales ofrecen dificultades a la hora de calcular el gasto calórico cuando se usan intensidades de ejercicio muy bajas o muy altas. Además, el precio de los mismos los hace poco accesibles. En resumen, se puede afirmar que no hay una forma determinada para obtener mediciones de alta exactitud al medir la actividad física y el gasto energético en los niños y las niñas. La naturaleza de los patrones de movimiento de ellos, la variedad de actividades que realizan y las limitaciones de cada método condicionan los resultados de las mediciones. Lo que se recomienda es la utilización de más de un método con el afán de triangular los resultados.

\section{BIBLIOGRAFÍA}

Bar-Or, O. (1994). Pediatric Sport Medicine for the Practitioner. New York: Springer.

Basset, D. (2000). Validity and reliability issues in objective monitoring of physical activity. RQES. $71,30-36$.

Bouten, C., Koekkoek, K., Verduin, M., Kodde, R. \& Janssen, J. ( 1997). A triaxial accelerometer and portable data processing unit for the assessment of daily physical activity. Transaction on Biomedical Engineering. 44, 136-147

Caspersen, C., Powell, K. \& Christensen, G. (1985). Physical activity, exercise and physical fitness. Public Health Reports. 100, 126-131.

Corbin, C., Pancrazi, R. \& Welk, G. (1994). Toward an understanding of appropriate physical levels for youth. Physical Activity and Fitness Research Digest. 1, 1-8.

Corbin, C. \& Pancrazi, R. (1996). How much physical activity is enough? JOPERD: 67, 33-39.

Craig, S., Coldberg, J \& Dietz, W. (1996). Psychosocial correlates of physical activity among fifth and eighth graders. Preventive Medicine. 25, 506-513.

Crocker, P., Bailey, D., Faulker, R., Kiwalski, K. \& McGrath, R. (1997). Measuring general levels of physical activity: preliminary evidence for the physical activity. Medicine and Science in Sports and Exercise. 29, 1344-1349.

Dale, D. (1999). Using the CSA activity monitors to determine if children compensate for restricted periods of physical activity. RQES. 68, 11-23. 
DuRant, R., Baranowski, T. Davis, H., Rhodes, T., Thompson, W. Greaves, K. \& Puhl, K (1993). Reliability and variability of indicators of heart rate monitoring in children. Medicine and Science in Sports and Exercise. 25, 389-395.

Eston, R., Rowland, A. \& Ingledew, D. (1998). Validity of heart rate, pedometry and accelerometry for predicting the energy cost of children's activities. Journal of Applied Physiology. 84, 362371.

Freedson, P., Melanson, E., \& Sirard, J. (1998). Calibration of the computer science and applications. Medicine and Science in Sports and Exercise. 30, 777-781.

Freedson, P., \& Miller, K. (2000). Objective monitoring of physical activity using motion sensors. RQES. 71, 21-29.

Going, S., Levin, S., Harrell, J., Stewart, D., Kushi, L., Cornell, C., Hunsberger, S., Corbin, C. \& Sallis, J. (1999). Physical activity assessment in American Indian schoolchildren in the Pathway Study. American Journal of Clinical Nutrition. 69, 788S-795S.

Gortmaker, S., Cheung, L., Peterson, K., Chomitz, G., Golditz, G., Field, A. \& Laird, N. (1999). Impact of a school-based interdisciplinary intervention on diet and physical activity among urban primary school children. Archives of Pediatric and Adolescent Medicine. 153, 975-983.

Janz, K. (1994). Validation of the CSA accelerometer for assessing children's physical activity. Medicine and Science in Sports and Exercise. 26, 369-375.

Kowalski, K., Crocker, R. \& Faulkner, R. (1997). Validation of the physical activity questionnaire for older children. Pediatric Exercise Science. 9, 174-186.

Mahoney, L., Lauer, R., Lee, J., \& Calrke, W. (1991). Factors affecting tracking of coronary heart disease risk factors in children. The muscative study. Journal of School Health. 61, 224-227.

Melanson, E. \& Freedson, P. (1996). Physical activity assessment: a review of methods. Critical Review in Food Science and Nutrition. 36, 385-396.

Montoye, H., Kemper, H., Saris, W., \& Washburn, R. (1996). Measuring Physical Activity and Energy Expenditure. Champaign: Human Kinetics.

Pancrazi, R., Corbin, C. y Welk, G. (2000). Physical activity for children and youth. JOPERD. 67, 38-43.

Pate, R. (1993). Physical activity assessment in children and adolescents. Critical Reviews in Food Science and Nutrition. 33, 321-326.

Pate, R., Baronowski, R, Dowda, M. y Trost, S. (1996). Tracking of physical activity in young children. Medicine and Science in Sports and Exercise. 28, 92-96.

Pate, R., Long, S., Dowda, M. Ott, A., Ward,. D., Saunders, R. (1999). Tracking of physical activity, physical inactivity, and health-related physical fitness in rural youth. Pediatric Exercise Science. 11, 364-373. 
Patterson, P. (2000). Reliability, validity, and methodological response to the assessment of physical activity via self-report. RQES. 71, 15-20.

Rippe, J., \& Hess, S. (1998). The role of physical activity in the prevention and management of obesity. Journal of the American Dietetic Association. 98, S31.

Rowland, T. \& Eston, R. (1997). Measurement of physical activity in children with particular reference to the use of heart rate and pedometry. Sports Medicine. 24, 258-272.

Rowland, T. (1998). The biological basis of physical activity. Medicine and Science in Sports and Exercise. 30, 392-399.

Sallis, J. (1991). Self-report measures of children's physical activity. Journal of School Health. 61, 215-219.

Sallis, J., Buono, M., Roby, J. Micale, F. \& Nelson, J. (1993). Seven-day recall and other physical activity self-reports in children and adolescents. Medicine and Science in Sports and Exercise. $25,99-108$.

Sallis, J. \& Saelens, B. (2000). Assessment of physical activity by self-report: Status, limitations and future directions. RQSE. 71, 1-12.

Trost, S., Pate, R., Dowda, M., Saunders, R., Ward, D. \& Felton, G. (1996). Gender differences in physical activity and determinants of physical activity in rural fifth grade children. Journal of School Health. 66, 145-150.

Trost, S., Ward, D. \& Burke, J. (1998). Validity of the computer science and application activity monitor in children. Medicine and Science in Sports and Exercise. 30, 629-933.

U.S. Department of Health and Human Services. (1996). Physical activity and health: reports of the

Surgeon General. Atlanta, GA: U.S Department of Health and Human Services, Centers for Disease Control and Prevention.

Welk, G. \& Corbin, C. (1995). The validity of the Tritrac- R3D activity monitor for the assessment of physical activity in children. RQES. 66, 202-209.

Welk, G. (2000). Measurement issues in the assessment of physical activity in children. RQES. 71, 59-73.

Welk, G. \& Wood, K. (2000). Physical activity assessments in physical education. JOPERD. 71, 30-40.

Weston, A., Petosa, R., \& Pate, R. (1997). Validation of an instrument for measurement for physical activity in youth. Medicine and Science in Sport and Exercise. 29, 138-143.

Whitaker, R., Wright, J., Pepe, M., Seidel, K. \& Dietz, W. (1997). Predicting obesity in young adulthood from childhood and parental obesity. The New England Journal of Medicine. 337, 869-873.

Fecha de recepción del artículo: 04 de agosto del 2004.

Fecha de aceptación del artículo: 23 de setiembre del 2004.

Fecha de publicación del artículo: 24 de setiembre del 2004. 